\title{
Research progress of berberine mediated photodynamic therapy (Review)
}

\author{
YA-WEN AN ${ }^{1}$, HONG-TAO JIN ${ }^{2}$, BO YUAN ${ }^{3}$, JIAN-CHUN WANG ${ }^{1}$, CHENG WANG $^{3}$ and HAN-QING LIU ${ }^{1}$ \\ ${ }^{1}$ Central Laboratory, Shenzhen Samii Medical Center, Shenzhen, Guangdong 518118; \\ ${ }^{2}$ Institute of Materia Medica, Chinese Academy of Medical Sciences and Peking Union Medical College, \\ Beijing 100050; ${ }^{3}$ Department of Neurology, Shenzhen Samii Medical Center, Shenzhen, Guangdong 518118, P.R. China
}

Received October 27, 2020; Accepted February 3, 2021

DOI: $10.3892 / \mathrm{ol} .2021 .12620$

\begin{abstract}
Berberine (BBR) is a plant secondary metabolite that has been used in photodynamic therapy (PDT) in the last few decades. The present review aimed to discuss the research progress of BBR-mediated photodynamic actions. The following key words were searched in several databases: 'Berberine' combined with 'photodynamic therapy', 'sonodynamic therapy (SDT)', 'ultraviolet', 'reactive oxygen' and 'singlet oxygen'. The results demonstrated that both type I and type II reactions participated in the photodynamic progression of BBR derivatives. In addition, the photochemical characteristics of BBR derivatives were affected by the polarity, $\mathrm{pH}$ and $\mathrm{O}_{2}$ content of solvents. DNA binding increases the lifespan of the photoexcited BBR state and generation of singlet oxygen $\left({ }^{1} \mathrm{O}_{2}\right)$. The chemical properties of substituents in different positions of the BBR skeleton are pivotal for its photochemical properties, particularly the methylenedioxy group at the C-2 and $\mathrm{C}-3$ positions. BBR is a promising agent for mediating both PDT- and SDT-treated diseases, particularly in tumors. However, further studies are required to validate their biological effects. In addition, the molecular mechanisms underlying the antitumor effects of BBR-PDT remain unclear and warrant further investigation. The structural modification and targeted
\end{abstract}

Correspondence to: Dr Han-Qing Liu, Central Laboratory, Shenzhen Samii Medical Center, 1 Jinniu West Road, Pingshan, Shenzhen, Guangdong 518118, P.R. China

E-mail: liuhanqing@ssmc-sz.com

Abbreviations: $\mathrm{BBR}$, berberine; ${ }^{3} \mathrm{BBR}^{*}$ : triple excited state of $\mathrm{BBR}$; $\mathrm{BBR}^{\circ+}$, BBR radical cation; PDT, photodynamic therapy; SDT, sonodynamic therapy; ROS, reactive oxygen species; ${ }^{1} \mathrm{O}_{2}$, singlet oxygen; $\mathrm{O}_{2}{ }^{-}$, superoxide radical anion; ${ }^{\circ} \mathrm{OH}$, hydroxyl radicals; DMF, 2,5-dimethyl furan; $\mathrm{CH}_{2} \mathrm{Cl}_{2}$, dichloromethane; EPR, electron paramagnetic resonance; $\mathrm{D}_{2} \mathrm{O}$, aqueous; $\mathrm{MeOH}$, methanol; DMSO, oxygenated dimethyl sulfoxide; Lys, lysozyme; EtOH, ethanol; EAC, ehrlich ascites carcinoma; PLGA, poly (lactic-co-glycolic acid); NPs, nanoparticles

Key words: berberine, photodynamic therapy, reactive oxygen species, target drug delivery delivery of BBR have made it possible to broaden its applications; however, experimental verification is required. Overall, BBR acts as a sensitizer for PDT and has promising development prospects.

\section{Contents}

1. Introduction

2. Photodynamic characteristics of BBR

3. Biological effects of BBR-mediated PDT

4. Targeted delivery for BBR

5. Conclusions and prospects

\section{Introduction}

Photodynamic therapy (PDT) has significantly developed in the last 100 years and is a promising therapeutic modality for the treatment of cancer, dermatosis and bacterial infections (1-3). The main components of PDT include a photosensitizer, molecular oxygen and light irradiation (1). Following light irradiation of the photosensitizer, molecular oxygen can produce reactive oxygen species (ROS), including singlet oxygen $\left({ }^{1} \mathrm{O}_{2}\right)$, superoxide radical anion $\left(\mathrm{O}_{2}{ }^{-}\right)$, hydrogen peroxide and hydroxyl radicals $\left({ }^{\circ} \mathrm{OH}\right)$, to exert various roles in different biological processes, in general, ${ }^{1} \mathrm{O}_{2}$ is involved in energy transfer and the free radicals are involved in electron transfers (4). The photosensitizer is the most important factor for PDT (2). However, cell specificity and selectivity remain insufficient for most photosensitizers (5). Notably, products from herbal plants, such as hypericin, curcumin, psoralen and berberine (BBR) have been demonstrated to act as photodynamic agents, exhibiting potential for wide applications in PDT (6-8).

BBR is an important isoquinoline alkaloid isolated from traditional medicines, such as Berberis aristata Sims, Coptis chinensis Franch and Coptis japonica Makino (Fig. 1). Coptis chinensis Franch belongs to the wild plant medicinal materials under secondary protection in China (9). BBR is produced in plants as a secondary metabolite and possesses a range of pharmacological properties (10-12). To the best of our knowledge, Philogène et al (13) were the first to demonstrate 
that BBR generates ${ }^{1} \mathrm{O}_{2}$, which is considered the main product of photodynamic reactions (2). Wang et al (14) studied the photodynamic activity of BBR on cancer cells in 1986. Our recent studies demonstrated that BBR-mediated PDT and sonodynamic therapy (SDT) significantly inhibit tumor cells in vivo $(15,16)$. As a photosensitizer, BBR possesses several advantages, such as easy production, low cost and various pharmacological effects, including antitumor functions (15). However, BBR also possesses several disadvantages, including toxicity and poor water solubility (16).

The present review focuses on the photodynamic characteristics of BBR and its biological effects via PDT and SDT, as well as its molecular mechanisms, defects and potential strategies to provide novel insights for future development.

\section{Photodynamic characteristics of BBR}

$\mathrm{BBR}$ is sensitive to light and can react in the presence of changing energy levels, such as several other secondary metabolites, including coralyne, sauguinarine and palmatine $(15,17)$. All plant compounds have specific biological functions, whose effects have been demonstrated to be effective in animal cells, including humans (17-23). Philogène et al (13) were the first to report that $\mathrm{BBR}$ is an ${ }^{1} \mathrm{O}_{2}$ generator via 2,5-dimethyl furan (DMF) experiments. DMF is easily oxidized, thus, by measuring its decrease in absorbance at $220 \mathrm{~nm},{ }^{1} \mathrm{O}_{2}$ generation can be indirectly measured (24). The results of this study demonstrated that the first-order rate constant $(k)$ of ${ }^{1} \mathrm{O}_{2}$ generation induced by BBR and UV was $2.1 \times 10^{-4} / \mathrm{S}^{1}$. Arnason et al (25) detected 1,270 nm near-infrared phosphorescence produced by BBR with near UV exposure, where the phosphorescence at $1,270 \mathrm{~nm}$ was considered as direct evidence of ${ }^{1} \mathrm{O}_{2}$ generation. The results of this study demonstrated that the singlet oxygen yield $\left(\Phi_{\Delta}\right)$ of BBR was 0.25 in dichloromethane $\left(\mathrm{CH}_{2} \mathrm{Cl}_{2}\right)$ solution. Since then, a series of studies have demonstrated that both type I and type II reactions are involved in UV light excitation for BBR, the photochemistry is influenced by polarity, oxygen content and $\mathrm{pH}$ of solvents, and the DNA-binding and photochemical functional group of BBR (26-28).

Types of photodynamic reactions for BBR. There are two types of reactions that are involved in PDT. Type I reactions involve electron transfers and result in the production of free radicals, while type II reactions involve energy transfer and result in the production of ${ }^{1} \mathrm{O}_{2}(4,5)$. The $1,270 \mathrm{~nm}$ near-infrared phosphorescence is considered the characteristic absorption peak of ${ }^{1} \mathrm{O}_{2}$ and has been used to calculate the $\Phi_{\Delta}$ in most studies $(29,30)$. The electron paramagnetic resonance (EPR) spin trapping technique is typically used to detect free radical photogeneration and triplet state, with the aid of 5,5-dimethyl-1-pyrroline N-oxide (26). EPR and 2,2,6,6-tetramethyl-4-piperidinol can also be used to detect ${ }^{1} \mathrm{O}_{2}(27)$.

Both types of reactions are involved in BBR-PDT (Fig. 2). Briefly, following exposure to UV light, BBR is excited (4). There are three ways for the excited-state BBR to return to the ground state: Internal conversion, release of fluorescence and reaching the triplet excited state of BBR ( $\left.{ }^{3} \mathrm{BBR}^{*}\right)$ through intersystem crossing (28). ${ }^{3} \mathrm{BBR}^{*}$ produces ${ }^{1} \mathrm{O}_{2}$ and other oxygen free radicals by energy transfer and electron transfer, under aerobic conditions (28). Both ${ }^{1} \mathrm{O}_{2}$ and free radicals can react with biomolecules, but ${ }^{1} \mathrm{O}_{2}$ is more efficient (2). Furthermore, a $1,270 \mathrm{~nm}$ phosphorescence can be detected in combination with ${ }^{1} \mathrm{O}_{2}$ generation (29).

Inbaraj et al (26) reported that BBR in aqueous $\left(\mathrm{D}_{2} \mathrm{O}\right)$ solutions exposed to light does not generate ${ }^{1} \mathrm{O}_{2}$. However, the $\Phi_{\Delta}$ in $\mathrm{CH}_{2} \mathrm{Cl}_{2}$ was 0.34 , which was higher than that detected in the study by Philogène et al (13); in non-polar environments, BBR can generate both ${ }^{1} \mathrm{O}_{2}$ and radical species, such as $\mathrm{O}_{2}{ }^{--}$, ${ }^{\circ} \mathrm{OH}$ and neutral BBR radicals (BBR'). Irradiation $(313 \mathrm{~nm})$ to $\mathrm{BBR}$ in frozen methanol $(\mathrm{MeOH})$ produced strong triplet signals, while in $\mathrm{D}_{2} \mathrm{O}$, no populated triplet state of BBR was observed (13). Brezová (27) et al demonstrated that both ${ }^{1} \mathrm{O}_{2}$ and $\mathrm{O}_{2}{ }^{--}$are produced following BBR irradiation in oxygenated dimethyl sulfoxide (DMSO) solvent. In addition, Jantová et al (31) observed the generation of superoxide anion radicals and ${ }^{1} \mathrm{O}_{2}$ in aerated solutions of BBR upon photoexcitation, and demonstrated that both type I and II mechanisms are involved in the efficient activation of molecular oxygen. Cheng et al $(28,32)$ reported that BBR can be activated by both 266 and $355 \mathrm{~nm}$ laser and produce ${ }^{3} \mathrm{BBR}^{*}$, BBR radical anions and $\mathrm{BBR}$ radical cations $\left(\mathrm{BBR}^{\circ+}\right)$. Furthermore, fast deprotonation of $\mathrm{BBR}^{\circ+}$ can produce BBR neutral radicals, suggesting that both type I and type II reactions are activated (28). These results were verified by investigating the interaction mechanisms between BBR and lysozyme (Lys), and the results demonstrated that type I reactions compete with ${ }^{1} \mathrm{O}_{2}(33)$. Shen and Ji (34) obtained the lowest triplet excitation energy of BBR and elucidated the characteristics of the triplet excited state using the time-dependent density functional theory. The results of this study proved that the direct energy transfer and electron transfer pathways are involved in the generation of ${ }^{1} \mathrm{O}_{2}$ and $\mathrm{O}_{2}{ }^{-}$, respectively. Liu and Zhang (35) investigated the UV-induced photo-electrocatalytic degradation of the BBR chloride form and demonstrated that the ROS produced during the reaction were $\mathrm{O}_{2}{ }^{-},{ }^{\circ} \mathrm{OH}$ and sulfate radicals.

Effects of solvents on the photodynamic reaction of $B B R$. It has been demonstrated that BBR can mediate photodynamic reactions. Several studies have confirmed that the photodynamic reactions of BBR are affected by several factors, such as solvent type, DNA binding and photochemical functional group $(28,34,36)$. The photochemical reactions of BBR are influenced by solvents polarity (26). Inbaraj et al (26) systemically studied the photochemistry of BBR in different solvents. The results of the present study demonstrated that the $\Phi_{\Delta}$ of BBR was the highest in $\mathrm{CH}_{2} \mathrm{Cl}_{2}(0.34)$, followed by toluene (0.044), 1,4-dioxane (0.042) and acetonitrile (MeCN; 0.038), while exposure to light did not generate ${ }^{1} \mathrm{O}_{2}$ in $\mathrm{D}_{2} \mathrm{O}$ solutions. The order of the fluorescence quantum yield in different solvents was as follows: 1,4-dioxane (0.071) $>$ zethanol $(\mathrm{EtOH}, 0.036)>\mathrm{MeCN}(0.025)>$ propylene carbonate (0.007). Taken together, these results suggest that BBR is a weak photosensitizer in water; however, it can produce both ${ }^{1} \mathrm{O}_{2}$ and radical species in nonpolar environments. Shen and Ji (34) observed the ROS photogeneration of BBR and demonstrated that both the generation of ${ }^{1} \mathrm{O}_{2}$ and $\mathrm{O}_{2}{ }^{-*}$ were highly dependent on solvent polarity. $\mathrm{O}_{2}{ }^{--}$was only produced in EtOH; however, ${ }^{1} \mathrm{O}_{2}$ was generated in both $\mathrm{EtOH}$ and benzene. Collectively, these results indicate that a 
A

B

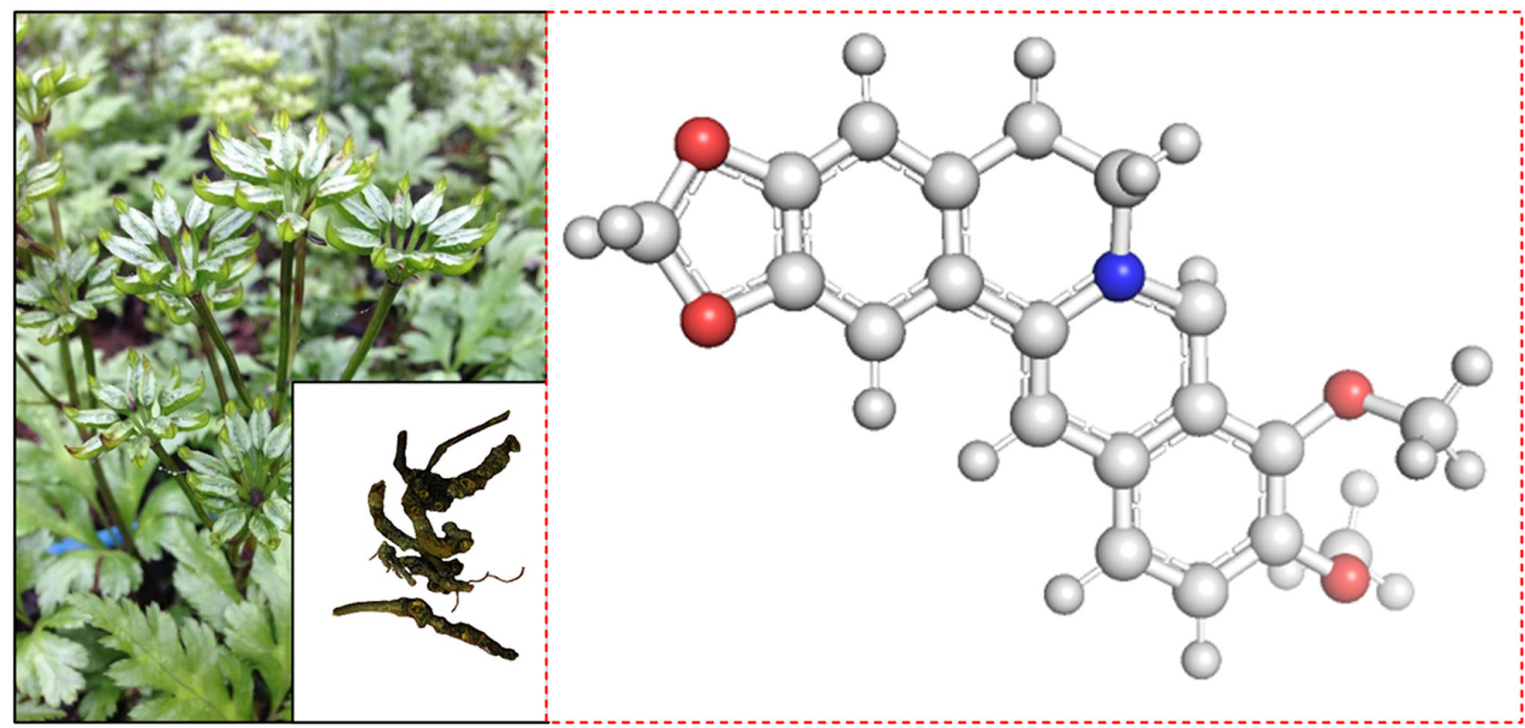

Figure 1. Coptis chinensis Franch and structural formula of BBR. (A) The root and stem of Coptis chinensis Franch. (B) Structural formula of BBR. BBR, berberine.

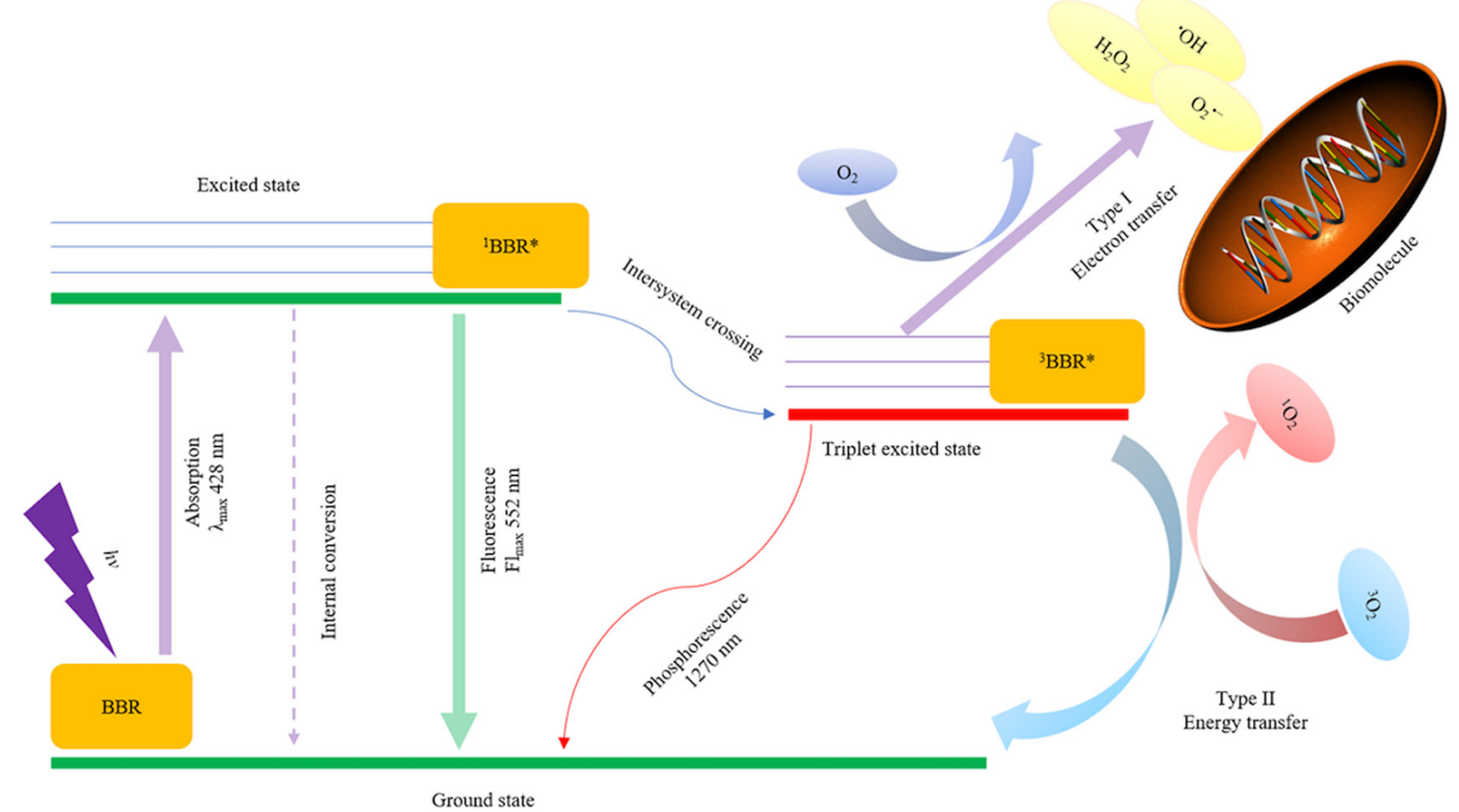

Figure 2. Graphical illustration of type I and type II photochemical reactions of BBR. The value of absorption and fluorescence was detected in MeCN. BBR, berberine; ' ${ }^{1} B B R *$, excited state of BBR; ${ }^{3} \mathrm{BBR}^{*}$, triple excited state of $\mathrm{BBR} ;{ }^{1} \mathrm{O}_{2}$, singlet oxygen; ${ }^{3} \mathrm{O}_{2}$, triplet oxygen; $\mathrm{O}_{2}{ }^{--}$, superoxide radical anion; $\mathrm{H}_{2} \mathrm{O}_{2}$, hydrogen peroxide; 'OH, hydroxyl radicals.

much higher $\Phi_{\Delta}$ is observed in non-polar solvents compared with polar solvents. Görner et al (37) implied that the decrease in BBR $\Phi_{\Delta}$ upon increasing solvent polarity was due to the dramatical acceleration of its internal conversion and reduction of the triplet rate formation yield. Notably, Cheng et al (28) reported that in laser flash photolysis, BBR exposure to a $266 \mathrm{~nm}$ laser in an aqueous solution produces hydrated electrons through a monophotonic process, and the hydrated electrons react with the steady state of BBR to form anion radicals, radical cations and neutral radicals. The quantum yield of photoionization was determined to be 0.03 . Notably, Görner et al (36) demonstrated that photoinduced electron ejection does not play a role in oxidation. Generally, BBR is more prone to photochemical reactions in nonpolar solvents than in polar solvents, and the ROS yield is higher in non-polar solvents $(28,36,37)$. In addition, the $\mathrm{pH}$ of solvents also affects the reaction (36). Görner et al (36) reported that the quantum yield of BBR-sensitized oxidation in oxygenated alkaline $\mathrm{MeOH}$ solution significantly increased with an increase in $\mathrm{pH}$, reaching a yield of 0.4 at $\mathrm{pH}$ 13.8. 
In addition to solvent polarity, the oxygen content of solvents is also considered to play an important role in the light activation of BBR. Brezová et al (27) demonstrated that upon irradiation, BBR produces both $\mathrm{O}_{2}{ }^{-}$and ${ }^{1} \mathrm{O}_{2}$ in DMSO. Furthermore, Hirakawa et al (38) reported that photoexcited BBR causes DNA cleavage under aerobic conditions, but no DNA photodamage was observed under anaerobic conditions. Jantová et al (31) revealed that EPR signals of photoexcited BBR in argon-saturated DMSO solutions are very low, while EPR signals of photoexcited BBR was higher in DMSO saturated by air or oxygen; the addition of SOD substantially decreased the formation of superoxide anion radicals, thus confirming unambiguously the photoinduced electron transfer from the photoexcited BBR to molecular oxygen; and the generation of superoxide anion adducts declined following the addition of superoxide dismutase; the aforementioned set of experiments confirmed the requirement of $\mathrm{BBR}, \mathrm{O}_{2}$ and light for effective ROS generation.

Notably, other reactions upon laser irradiation of BBR exist besides photooxidation processes. Cheng et al $(28,32,33)$ studied the photoionization and photoexcitation of BBR in $\mathrm{N}_{2}$ - and $\mathrm{O}_{2}$-saturated systems. The results of these studies demonstrated that upon laser excitation at $355 \mathrm{~nm}$, an absorption band $\sim 420 \mathrm{~nm}$ and an obvious bleach $\sim 360 \mathrm{~nm}$ of BBR was observed in $\mathrm{N}_{2}$-saturated $\mathrm{MeCN}$, and $\mathrm{O}_{2}$ significantly affected the species with $420 \mathrm{~nm}$ absorption. In the presence of $\mathrm{O}_{2}$, the decay of ${ }^{3} \mathrm{BBR}^{*}$ in $\mathrm{N}_{2}$-bubbled $\mathrm{MeCN}$ was accelerated; the absorption value of bleaching is lower in the $\mathrm{O}_{2}$-saturated system than in the $\mathrm{N}_{2}$-saturated system, which indicates that another transient species may have been formed in addition to ${ }^{3} \mathrm{BBR}^{*}$, and the total yield of bleaching should include both $\mathrm{BBR}^{\cdot+}$ and ${ }^{3} \mathrm{BBR}^{*}$ in the $\mathrm{N}_{2}$-saturated system (32). Similar phenomena were observed upon $266 \mathrm{~nm}$ laser excitation. After the laser pulse, the depletion of ground states of BBR was observed as chartered absorption bleaching at $350 \mathrm{~nm}$ and both ionization and excitation contributed to the bleaching of BBR in the $\mathrm{N}_{2}$-saturated system, while in the $\mathrm{O}_{2}$-saturated system, only ionized processes were involved (28). Active radicals of BBR have also been observed, induced by laser oxidized tryptophan (Trp) of Lys via electron transfer progression in $\mathrm{O}_{2}$-free polar solvents; however, in the presence of $\mathrm{O}_{2}$, both electron and energy transfer mechanisms are involved in photooxidation (33). Görner et al (37) studied the photoreduction and ketone-sensitized reduction of alkaloids, including BBR. The results of this study demonstrated that the major photoproducts of alkaloids are dihydroalkaloids and are reverted back to the corresponding alkaloids upon exposure of $\mathrm{O}_{2}$. In addition, it was indicated that Rose Bengal, a photosensitizer, significantly accelerates BBR photooxidation in oxygenated alkaline $\mathrm{MeOH}$ solutions, suggesting the importance of Rose Bengal-sensitized ${ }^{1} \mathrm{O}_{2}$ in the reaction $(36,37)$.

Taken together, these findings demonstrate the complexity of the photochemical reactions of BBR and the important roles of $\mathrm{O}_{2}$. Since ${ }^{1} \mathrm{O}_{2}$ has proven efficient in destroying biomolecules, more attention should be paid on the balance between photooxidation, photoionization and photoreduction in biological applications.

Interaction between $B B R$ and DNA. DNA is an important molecular target of BBR (39). DNA binding and its effects on the photochemical properties of BBR were recently discovered. Hirakawa et al (38) revealed the mechanism of guanine-specific photooxidation of BBR and demonstrated that BBR easily binds to DNA and forms a strong fluorescent complex, which is necessary for ${ }^{1} \mathrm{O}_{2}$ generation. In addition, BBR is unable to produce ${ }^{1} \mathrm{O}_{2}$ in the absence of DNA. Furthermore, the authors discovered switches in ${ }^{1} \mathrm{O}_{2}$ activity resulting from the DNA microenvironment, and that BBR can bind to DNA by electrostatic forces. This binding significantly enhances the lifespan of its photoexcited state by inhibiting quenching via electrostatic forces (40). The dynamics of ${ }^{1} \mathrm{O}_{2}$ generation by DNA-binding BBR were assessed and the results demonstrated that ${ }^{1} \mathrm{O}_{2}$ generation is led by energy transfer from the DNA-binding BBR complex to molecular oxygen (41). The guanine sequence decreased the $\Phi_{\Delta}$ by deactivating the singlet excited state of BBR, and steric hindrance of DNA decreased the constant rate of ${ }^{1} \mathrm{O}_{2}$ generation (41).

BBR also induces DNA photodamage (40). Guanine is the most easily oxidized base of DNA (42). Hirakawa et al (38) demonstrated that photoexcited BBR induces piperidine-sensitive calf thymus DNA damage, specifically at almost all guanine residues under aerobic conditions, but barely induces direct DNA strand breaks. Conversely, Jantová et al (31) reported that BBR significantly induces direct DNA strand breaks in NIH-3T3 and Ehrlich ascites carcinoma (EAC) cells. Cheng et al (43) demonstrated the capability of BBR to photosensitize DNA cleavage, and demonstrated that electron transfer via BBR neutral radicals to guanine neutral radicals plays an important role in DNA cleavage, and that both single- and double-stranded DNA at guanine moieties can be selectively cleaved by photoexcited BBR. The results of this study also clarified the molecular mechanisms of BBR-induced photodamage of dGMP and DNA.

DNA binding enhances the lifespan of photoexcited BBR states and generates ${ }^{1} \mathrm{O}_{2}$; meanwhile, it is destroyed in the process $(31,38)$. Understanding and utilizing this interaction and antinomy may provide insight to photosensitizer design based on BBR and its biological applications.

Photochemical functional groups of BBR. The functional groups of BBR are important for its photochemical properties $(27,44,45)$. The structures of BBR derivatives are summarized in Fig. 3. Brezová et al (27) compared the photoexcited oxygen activation of BBR and its derivatives, palmatine and jatrorrhizine. The methylenedioxy group at the C-2 and C-3 positions of BBR is photolabile (27). When these groups are replaced by two methoxy groups (palmatine) or C-2 methoxyl and C-3 hydroxyl (jatrorrhizine), the photochemical generation of $\mathrm{O}_{2}{ }^{\cdot-}$ and ${ }^{1} \mathrm{O}_{2}$ significantly decreases in DMSO solution (27). The UV/Vis absorption in regions of 350 and $425 \mathrm{~nm}$ in DMSO and EtOH is also influenced by the substitutional changes (27). However, DNA binding and associated photochemical reactions have failed to demonstrate differences between BBR and palmatine $(38,40,41)$. In addition, the effects of solvent polarity on the photoirradiation reactions of BBR and palmatine are similar (37). The antitumor effects of palmatine mediated PDT were proven in vitro using several cancer cell lines, such as MCF-7 and HT-29 cells $(44,46,47)$.

Coralyne, a protoberberine alkaloid (45), has a C-7 methyl group replace based on palmatine, which has been 


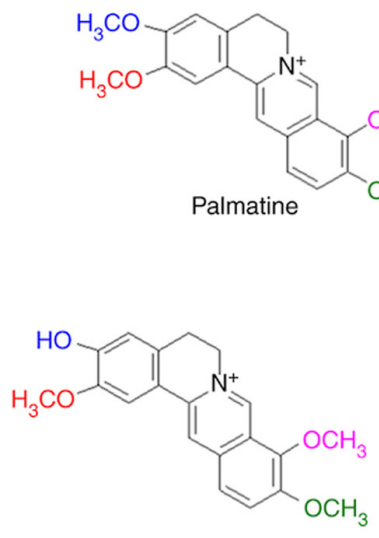

Jatrorrhizine

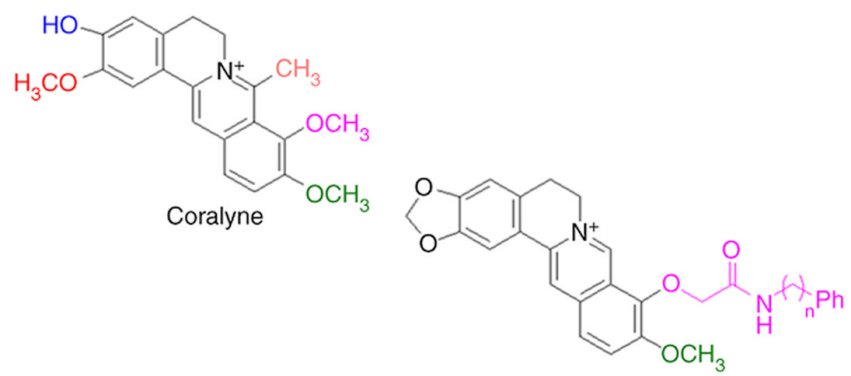

9-O-substitruted analogs-1

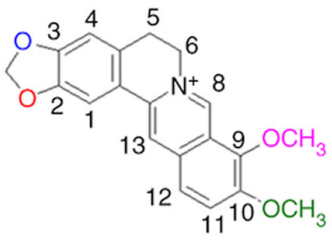

BBR

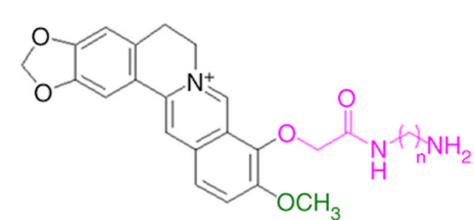

9-O-substitruted analogs-2

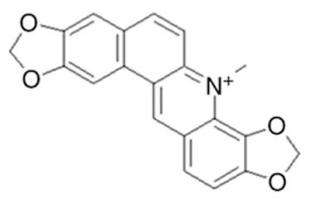

Sauguinarine

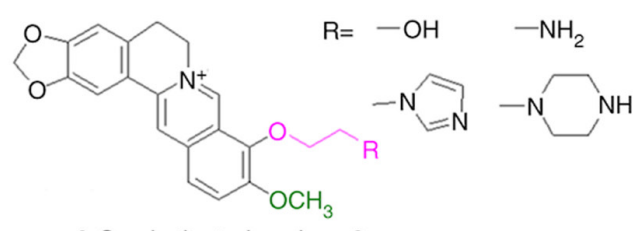

9-O-substitruted analogs-3

Figure 3. Effects of functional groups on the photoactivities of BBR. Chemical properties of substituents in different positions of the BBR skeleton are pivotal for its photochemical properties, particularly the methylenedioxy group at C-2 and C-3 positions, C-7 methyl group and the 9-O-position of the isoquinoline chromophore. BBR, berberine.

demonstrated to cause robust photosensitization of cancer cells (45). The $\Phi_{\Delta}$ of coralyne is independent of solvent polarity unlike BBR and palmatine (37). Patro et al (48) reported that coralyne induces significant nicking of plasmid DNA via an $\mathrm{O}_{2}$-independent photochemical process; however, the phenomenon has not been observed in BBR or jatrorrhizine. Ihmels and Salbach (49) demonstrated that coralyne irradiation results in significant DNA damage. The extent of the damage does not differ between aerobic and anaerobic conditions; however, the extent of the damage is significantly different to that induced by BBR.

Basu et al $(50,51)$ synthesized BBR analogs with substituents at the 9-O-position of the isoquinoline chromophore and demonstrated that these analogs enhance the binding affinities of BBR to both DNA and double stranded poly(A). Liu et al (52) confirmed increased DNA binding of several 9-O-substituted $\mathrm{BBR}$ derivatives, and one of the derivatives exhibited higher cytotoxicity in HL-60 and BGC-823 cells compared with BBR. Since the photochemical functional groups were not altered, and DNA binding has significant influence on the $\Phi_{\Delta}$ of BBR, such substitutions may enhance the photochemical properties of BBR; however, further experimental verification is required. Arnason et al (25) determined the $\Phi_{\Delta}$ of sanguinarine, which was demonstrated to be lower than BBR (0.16 vs. 0.25$)$. The median lethal dose of sanguinarine to mosquito larvae significantly decreased from $23.3 \mathrm{mg} / \mathrm{ml}$ to $0.096 \mathrm{mg} / \mathrm{ml}$ following near-UV exposure. Görner et al (36) confirmed that the absorption spectra of sanguinarine is altered upon change in $\mathrm{pH}$ in a 9:1 mixture of $\mathrm{MeOH}$ and water, whereby the main product upon irradiation at $313 \mathrm{~nm}$ is 6-oxysanguinarine. The $\Phi_{\Delta}$ of sanguinarine is lower than BBR in several solvents (37), suggesting that the methylenedioxy group of C-9 and C-10 is not beneficial to photoactivity.

Taken together, these results suggest that changing the substituents of photochemical functional groups of BBR affect its photochemical properties, which provides insight for the molecular design of a BBR-based photosensitizer.

\section{Biological effects of BBR-mediated PDT}

Since the discovery of the photochemical properties of BBR, the applications of BBR- mediated PDT in biology have attracted great attention.

Application of BBR-mediated-PDT in non-neoplastic diseases. BBR-PDT has been proven to treat a variety of non-neoplastic diseases. Philogène et al (13) demonstrated the phototoxicity of BBR to mosquito larvae, whereby the median lethal concentration decreased from 250 to $8.8 \mathrm{ppm}$ in the presence of near UV. Molero et al (53) reported that the growth of Allium cepa Linn. var. cepa roots is inhibited by BBR upon irradiation of violet light $(420 \mathrm{~nm})$. Inbaraj et al (26) determined the phototoxicity of BBR to $\mathrm{HaCaT}$ keratinocytes and demonstrated that $50 \mu \mathrm{M}$ BBR combined with UVA irradiation decreases the cell viability to $20 \%$, and the DNA damage exhibited a 3-fold increase compared with UVA alone. BBR is an efficient fluorescent probe, and BBR-PDT has demonstrated potent bactericidal effects, both in vivo and 


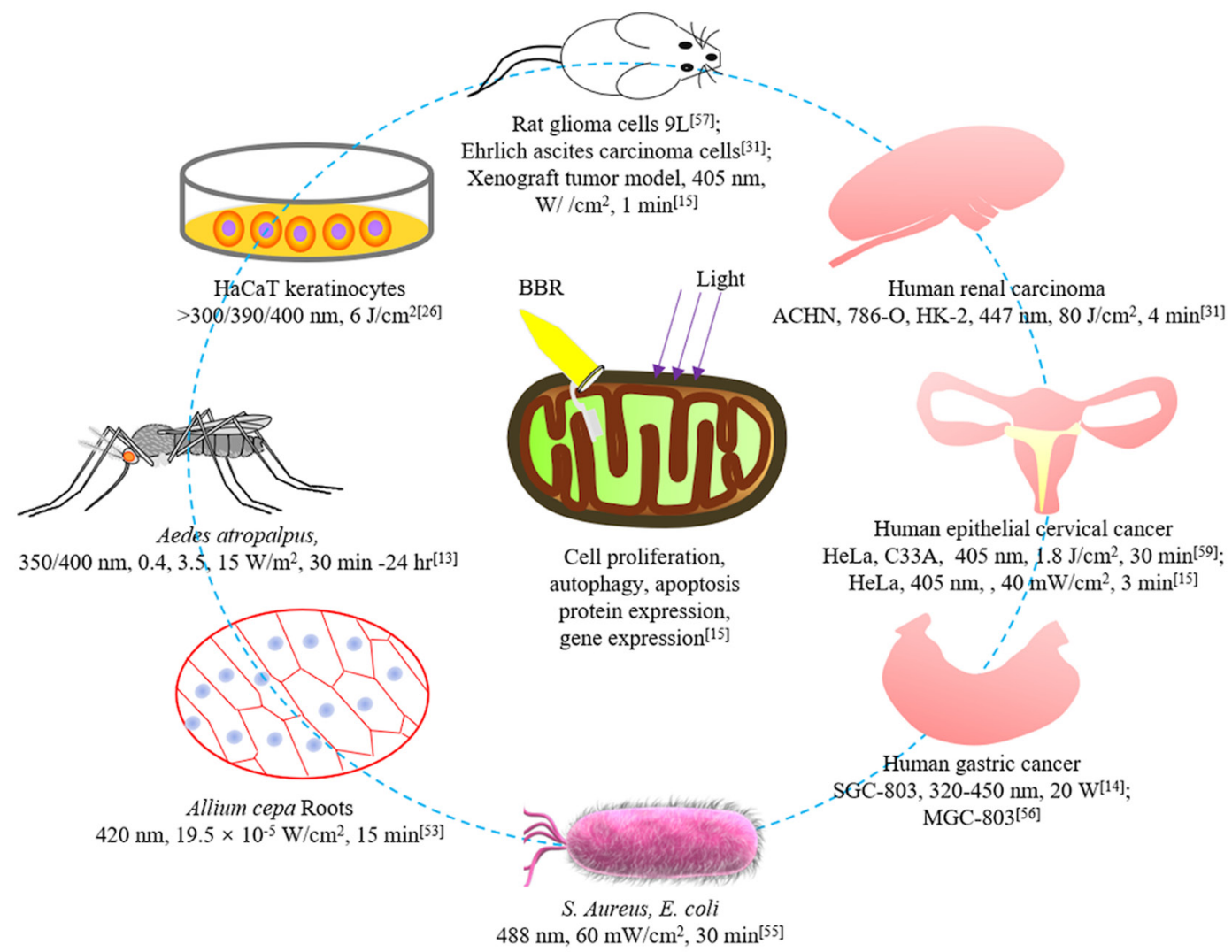

Figure 4. Biological effects of BBR-PDT. BBR-PDT exhibits destructive effects in different cells, including human tumor cells. The illumination parameters involved in relevant studies are presented. BBR, berberine; PDT, photodynamic therapy.

in vitro (54). Lee et al (55) reported that BBR successfully stains the Gram-positive bacteria, Staphylococcus aureus (S. aureus), whereas it only partly stains the Gram-negative Escherichia coli (E. coli). BBR-PDT significantly decreases the viability of $S$. aureus, and the bactericidal effects occur in a dose-dependent manner (5-10 mM BBR). BBR-PDT also exhibits bactericidal effects in $E$. coli; however, the efficiency is lower and the required concentration of BBR is higher (55).

Antitumor effects of BBR-mediated PDT. Recently, the antitumor effects of BBR-PDT have attracted great interest. The activity in vitro was first demonstrated by Wang in 1986 (14), who observed the strong inhibitory proliferation of SGC-803 gastric cancer cells combined with BBR $(10 \mu \mathrm{g} / \mathrm{ml})$ under illumination, using a black light lamp (320-450 nm; $20 \mathrm{~W})$. Ma et al (56) demonstrated that BBR combined with 5-ALA-PDT significantly inhibits the proliferation of MGC-803 gastric cancer cells, induces cell apoptosis, inhibits the expression levels of survivin and $\mathrm{Bcl}-2$, and upregulates the expression levels of p53 and Bax. Taken together, these results suggest that BBR has the potential to be used in combination with other photosensitizers. Chen et al (57) studied the effects of BBR in combination with argon ion lasers on 9L rat glioma cells, and demonstrated that the argon ion laser enhances the inhibition of BBR on DNA, RNA and protein biosynthesis, resulting in cell-killing effects. Jantová et al (31) compared the cytotoxic and phototoxic effects on murine fibroblast NIH-3T3 and EAC cells. The results demonstrated that EAC cells are more sensitive to photoactivated BBR compared with non-cancer NIH-3T3 cells. BBR-PDT induces the DNA damage of EAC cells, subsequently blocking the $\mathrm{S}$ and $\mathrm{G}_{2} / \mathrm{M}$ phases, and decreases cell proliferation. In addition, DNA damage induces necrotic or apoptotic death of EAC cells (31). Lopes et al (58) demonstrated that BBR cytotoxicity increases by $20 \%$ following the combination of light. ROS production and activation of autophagy and apoptosis induced by BBR-PDT are responsible for the death of renal carcinoma cells. In addition, BBR-PDT affects the expression of anticancer drug target genes in 786-O cells (58). Warowicka et al (59) investigated the potential anticancer effects of a protoberberine alkaloidal fraction on HeLa and C33A cervical cancer cells. Liu et al (15) verified the antitumor effects of BBR-PDT in xenograft animal models, and revealed the role of the PI3K/AKT/mTOR signaling pathway.

The biological effects of BBR-PDT are summarized in Fig. 4. Among these, the mechanisms of BBR-PDT in treating tumors may involve inhibiting cell proliferation, inducing apoptosis and autophagy, and altering the expression of proteins or genes (15). However, there are a few studies currently investigating these mechanisms. BBR exhibits clear inhibitory effects on different types of cancer, including colorectal, lung, ovarian, prostate, liver and cervical cancers (60). This suggests that BBR-PDT may have more potent antitumor effects compared with other photosensitizers due to the superposition of the antitumor effects of BBR and PDT $(15,61)$. However, only a few studies have proven the antitumor effects of BBR-PDT $(15,31,59)$. The possible reasons are as follows (16): i) There are multiple factors influencing the photodynamic 
reactions of BBR; ii) the hydrophobic properties, along with poor stability and bioavailability of BBR limits its application; iii) BBR has high toxicity, particularly when administered by injection; and iv) the light source of BBR-PDT is UV, which has several side effects. such as burn and pain and no advantages compared with other photosensitizers (2).

$B B R$-mediated SDT. SDT is a promising new option for the invasive treatment of tumors (62). SDT is similar to PDT in that it requires the combination of sensitizers, ultrasound and molecular oxygen (61). The major advantage of SDT compared with PDT is the increased penetration of ultrasound through mammalian tissues compared with light, indicating that it can be used to treat deeper and less accessible tumors $(61,62)$. As the light source of BBR-PDT is UV, which has several side effects, such as burn and pain, BBR-SDT may be a more favorable choice for treating tumors (16). Wang et al (63) reported that $\mathrm{BBR}$ can enhance ultrasound-induced BSA molecule damage. The increase in the degree of damage is accompanied with the increase of BBR concentration and ultrasonic irradiation time. It was further revealed that BBR-SDT-mediated BSA damage is mediated by the production of ROS, particularly ${ }^{1} \mathrm{O}_{2}(63)$.

Atherosclerosis (AS) is the major contributing factor of cardiovascular events. SDT-targeting AS is considered a novel promising treatment strategy for AS (64). Tian and Guo (65) investigated the effects of BBR-SDT on macrophages within atherosclerotic plaques. Kou et al (66) demonstrated that BBR-SDT induces autophagy and cholesterol efflux in THP-1 macrophages and derived foam cells, which may potentially involve inhibiting phosphorylation of the PI3K/AKT/mTOR signaling pathway. In addition, the effects of BBR-mediated SDT on macrophages within atherosclerotic plaques were investigated.

Only a few studies have reported the antitumor effects of BBR-SDT. For example, Liu et al (16) demonstrated that BBR-SDT inhibits the proliferation of HeLa cells and induces apoptosis both in vitro and in vivo, and revealed the role of the $\mathrm{PI} 3 \mathrm{~K} / \mathrm{AKT} / \mathrm{mTOR}$ signaling pathway in this process. However, further studies are required to validate the antitumor effects of BBR-SDT. SDT has several advantages over PDT due to its deeper penetration and fewer side effects (64). However, the chemical properties of BBR, such as its hydrophobic properties, poor stability and bioavailability, and high toxicity need to be resolved (16).

\section{Targeted delivery for BBR}

The question remains as to how to overcome the physical shortcomings of BBR. Feasible options include chemical structure modification and targeted drug delivery $(51,67)$. The photochemical properties of BBR are associated with the substituents at the C-2, C-3, C-7 and 9-O positions $(27,45,50)$. Appropriate chemical structure modifications may increase its water solubility and retain or even improve its photodynamic activity; however, this requires experimental verification (68). In addition, targeted delivery may also avoid the dissolution and low absorption of BBR (69-73).

Several nanoparticulate delivery systems have been used to target the delivery of BBR. For example, Sun and Ouyang (67) prepared BBR hydrochloride, a nanoemulsion, which is a clear transparent solution with stable contents and diameter, even under high humidity and high temperatures. As a stable drug delivery system, BBR nanoemulsion has a high affinity for gastrointestinal lymphatic tissue. It can deliver larger amounts of BBR into the blood via the lymphatic system, avoid the biotransformation and absorption of the liver and intestines, and exhibit a first pass effect in the liver (67). Solid lipid nanoparticles can effectively control drug release, avoid drug degradation or leakage, and have potent targeting properties (74). Hou and Zhou (75) prepared solid lipid nanoparticles of BBR hydrochlorideusing a rotary-evaporated film-ultrasonication method. In addition, Wang et al (76) determined its entrapment efficiency.

Liposomes have similar structures to cell membranes and high affinities to tissues or cells (77). There are several approaches for the preparation of BBR liposomes, such as thin film evaporation and active loading (78). Incubation temperature and duration, the proportional weight of drugs and lipids, soybean phosphatidylcholine and cholesterol affect the encapsulation rate (79). Luo et al (78) prepared a long-circulating liposome of BBR hydrochloride using an ionophore A23187-mediated ZnSO4 gradient method. Using poly (lactic-co-glycolic acid) (PLGA) nanoparticles is a new strategy to promote specific targeting and elimination of drugs (80). Khan et al (81) developed a co-delivery system of BBR and doxorubicin using the conjugation/encapsulation strategy through PLGA nanoparticles. Co-delivery of BBR and other anticancer drugs is valuable to achieve higher anticancer effectivity (82). Guo et al (83) designed a liver-target nanotechnology system to facilitate BBR consisting of $\alpha$-tocopheryl hydrophobic core and on-site detachable polyethylene glycol-thiol shell to improve its liver deposition.

Cellular and molecular targeting can further enhance the effects of BBR and decrease its adverse reactions. Bhatnagar etal (84)preparedBBR chloride(BRB)-encapsulated hyaluronic acid-grafted [d(HA)-g]-PLGA nanoparticles (NPs). The BRB-d(HA)-g-PLGA NPs were used to target CD44-positive cancer cells. These NPs released BRB at a faster rate in HeLa and MCF-7 cells compared with BRB-PLGA NPs and bulk BRB, and increased its cytotoxicity. These results offer a promising and improved alternative for antitumor therapy. Song et al (68) obtained a novel mitochondria-targeting nanodrug self-assembled BBR derivative from a 9-O-octadecyl substitute using a simple nano-precipitation approach. These authors further used 1,2-distearoyl-sn-glycero-3-phosphoethanolamine-N-[methoxy (polyethyleneglycol) 2000] to modify the BBR derivative to increase its stability. Negatively charged HA was further coated to conceal positive charges and achieve tumor targeting. Grebinyk et al (85) formed a nanocomplex combining $\mathrm{C}_{60}$ fullerene and $\mathrm{BBR}$, and $\mathrm{C}_{60}$ was demonstrated to promote $\mathrm{BBR}$ intracellular uptake by leukemic cells.

Collectively, these targeted delivery strategies provide solutions to the limitations of BBR, such as its hydrophobic properties, poor stability and bioavailability. Cellular and molecular targeted delivery allows the possibility of further improving its therapeutic effects. However, there is currently no evidence regarding the use of these BBR delivery systems in PDT or SDT, which is worth investigating in prospective studies. In addition, the toxicity of BBR should be concerned 
in future applications with targeted delivery technology, particularly for free BBR, which may produce systemic adverse reactions.

\section{Conclusions and prospects}

BBR is a photosensitizer that can be activated by both light and ultrasound, and produce ${ }^{1} \mathrm{O}_{2}$. Both type I and type II reactions participate in the photochemical reactions of BBR. However, BBR reactions under ultrasound irradiation remain unclear. Both BBR-PDT and BBR-SDT have been proven to inhibit the proliferation and induce the apoptosis of several tumor cell lines in vitro. However, only a few studies have been validated in animal models, and the antitumor activities in vivo require further confirmation. Notably, both BBR-PDT and BBR-SDT can induce autophagy, which may be associated with the phosphorylation inhibition of the PI3K/AKT/mTOR signaling pathway. Although autophagy is generally considered to exert protective effects on cells, its role in the biological effects of BBR-PDT and BBR-SDT remain to be elucidated, particularly their antitumor effects. In addition, only a few proteins and genes involved in apoptosis have been identified to be regulated by BBR-PDT or BBR-SDT. Thus, it is important to clarify the antitumor mechanisms of BBR-PDT and BBR-SDT in prospective studies.

The photothermic characteristics of BBR are affected by polarity, $\mathrm{pH}$ and $\mathrm{O}_{2}$ content of solvents. Its hydrophobic properties, along with poor stability and bioavailability, and the high toxicity of BBR limits its application. It is also unclear whether the interactions between BBR and DNA is beneficial for PDT. Appropriate structural modifications at the C-2, C-3, C-7 and 9-O positions may change the water solubility and photodynamic efficiency of BBR; however, whether this is advantageous to BBR-PDT requires experimental verification. Targeting drug delivery via microbubbles may bypass the physical and chemical defects of BBR and enhance its antitumor effects via PDT and SDT. However, it is worth noting that BBR is highly toxic following intravenous injection. Although targeted delivery can theoretically only sequester BBR in the target tissues, the safety requires verification, particularly with regards to systemic toxicity.

In conclusion, BBR has a developmental prospect as a sensitizer for PDT and SDT treatment. Its simple structure, low cost and extensive pharmacological activities, such as antitumor effect, exhibit great advantage over other photosensitizers. Thus, the present study hypothesizes that BBR-PDT and BBR-SDT will be extensively used in clinical treatment in the future.

\section{Acknowledgements}

Not applicable.

\section{Funding}

The present review was supported by the Shenzhen Science and Technology Planning Project (grant nos. JCYJ20180302153611416 and JYCJ20190808160011529) and the Research Foundation of Shenzhen Samii Medical Center.

\section{Availability of data and materials}

Not applicable.

\section{Authors' contributions}

YWA and HQL drafted the initial manuscript. YWA, HTJ and HQL confirmed the authenticity of all the raw data. HTJ, BY, JCW and CW performed the literature review and produced Figs. 1 and 4. All authors have read and approved the final manuscript.

\section{Ethics approval and consent to participate}

Not applicable.

\section{Patient consent for publication}

Not applicable.

\section{Competing interests}

The authors declare that they have no competing interests.

\section{References}

1. Rkein AM and Ozog DM: Photodynamic therapy. Dermatol Clin 32: 415-425, 2014.

2. Chilakamarthi U and Giribabu L: Photodynamic therapy: Past, present and future. Chem Rec 17: 775-802, 2017.

3. Moy LS, Frost D and Moy S: Photodynamic therapy for photodamage, actinic keratosis, and acne in the cosmetic practice. Facial Plast Surg Clin North Am 28: 135-148, 2020.

4. Ghorbani J, Rahban D, Aghamiri S, Teymouri A and Bahador A: Photosensitizers in antibacterial photodynamic therapy: An overview. Laser Ther 27: 293-302, 2018.

5. Zhang J, Jiang C, Figueiró Longo JP, Azevedo RB, Zhang H and Muehlmann LA: An updated overview on the development of new photosensitizers for anticancer photodynamic therapy. Acta Pharm Sin B 8: 137-146, 2018.

6. Abrahamse $\mathrm{H}$ and Hamblin MR: New photosensitizers for photodynamic therapy. Biochem J 473: 347-364, 2016.

7. Kataoka H, Nishie H, Hayashi N, Tanaka M, Nomoto A, Yano S and Joh T: New photodynamic therapy with next-generation photosensitizers. Ann Transl Med 5: 183, 2017.

8. Mansoori B, Mohammadi A, Amin Doustvandi M, Mohammadnejad F, Kamari F, Gjerstorff MF, Baradaran B and Hamblin MR: Photodynamic therapy for cancer: Role of natural products. Photodiagnosis Photodyn Ther 26: 395-404, 2019.

9. Ortiz LM, Lombardi P, Tillhon M and Scovassi AI: Berberine, an epiphany against cancer. Molecules 19: 12349-12367, 2014.

10. Neag MA, Mocan A, Echeverria J, Pop RM, Bocsan CI, Crişan G and Buzoianu AD: Berberine: Botanical occurrence, traditional uses, extraction methods, and relevance in cardiovascular, metabolic, hepatic, and renal disorders. Front Pharmacol 9: 557, 2018.

11. Feng X, Sureda A, Jafari S, Memariani Z, Tewari D, Annunziata G, Barrea L, Hassan STS, Smejkal K, Malaník M, et al: Berberine in cardiovascular and metabolic diseases: From mechanisms to therapeutics. Theranostics 9: 1923-1951, 2019.

12. Liu D, Meng X, Wu D, Qiu Z and Luo H: A natural isoquinoline alkaloid with antitumor activity: Studies of the biological activities of berberine. Front Pharmacol 10: 9, 2019.

13. Philogène BJ, Arnason JT, Towers GH, Abramowski Z, Campos F, Champagne D and McLachlan D: Berberine: A naturally occurring phototoxic alkaloid. J Chem Ecol 10: 115-123, 1984.

14. Wang MX, Huo LM, Yang HC, Gao YJ and E Z: An experimental study on the photodynamic activity of berberine in vitro on cancer cells. J Tradit Chin Med 6: 125-127, 1986.

15. Liu HQ, An YW, Hu AZ, Li MH and Cui GH: Photodynamic therapy enhanced the antitumor effects of berberine on HeLa cells. Open Chemistry 17: 413-421, 2019. 
16. Liu H, Zheng T, Zhou Z, Hu A, Li M, Zhang Z, Yu G, Feng H, An Y, Peng J and Chen Y: Berberine nanoparticles for promising sonodynamic therapy of a HeLa xenograft tumour. Rsc Advances 9: 10528-10535, 2019.

17. Acamovic T and Brooker JD: Biochemistry of plant secondary metabolites and their effects in animals. Proc Nutr Soc 64 403-412, 2005

18. Schreiner M, Mewis I, Huyskens-Keil S, Jansen MAK, Zrenner R Winkler JB, O'Brien N and Krumbein A: UV-B-induced secondary plant metabolites-potential benefits for plant and human health. Crit Rev Plant Sci 31: 229-240, 2012.

19. Gismondi A, Marco GD, Canuti L and Canini A: Antiradical activity of phenolic metabolites extracted from grapes of white and red Vitis vinifera L. cultivars. Vitis J Grapevine Res 56 19-26, 2017.

20. Makkar HPS, Siddhuraju P and Becker K: Plant Secondary Metabolites. Humana Press, Totowa, NJ, USA, 2007.

21. Lockwood B: Plant Secondary Metabolites Occurrence, Structure and Role in the Human Diet. Crozier A, Clifford MN and Ashihara H, (eds). Blackwell Publishing Ltd. pp384. GBP 99.50, ISBN 13: 978-1-4051-2509-3. Phytochemistry 69: 1288-1288, 2008.

22. Steglich W: Plant Secondary Metabolites. Occurrence, Structure and Role in the Human Diet. Edited by Alan Crozier, Clifford MN and Ashihara $\mathrm{H}$, (eds). Angewandte Chemie International Edition 46: 8113-8114, 2007

23. Crozier A, Clifford MN and Ashihara H: Plant Secondary Metabolites: Occurrence, Structure and Role in the Human Diet. Blackwell Publishing Ltd. UK, 2006.

24. Agarwal R, Zaidi SI, Athar M, Bickers DR and Mukhtar $\mathrm{H}$ Photodynamic effects of chloroaluminum phthalocyanine tetrasulfonate are mediated by singlet oxygen: In vivo and in vitro studies utilizing hepatic microsomes as a model membrane source. Arch Biochem Biophys 294: 30-37, 1992.

25. Arnason JT, Guerin B, Kraml MM, Mehta B, Redmond RW and Scaiano JC: Phototoxic and photochemical properties of sanguinarine. Photochem Photobiol 55: 35-38, 1992.

26. Inbaraj JJ, Kukielczak BM, Bilski P, Sandvik SL and Chignell CF: Photochemistry and photocytotoxicity of alkaloids from Goldenseal (Hydrastis canadensis L.) 1. Berberine. Chem Res Toxicol 14: 1529-1534, 2001.

27. Brezová V, Dvoranova D and Kost'alova D: Oxygen activation by photoexcited protoberberinium alkaloids from Mahonia aquifolium. Phytother Res 18: 640-646, 2004.

28. Cheng LL, Wang M, Zhu H, Li K, Zhu RR, Sun XY, Yao SD, Wu QS and Wang SL: Characterization of the transient species generated by the photoionization of Berberine: A laser flash photolysis study. Spectrochim Acta A Mol Biomol Spectrosc 73: 955-959, 2009.

29. Hackbarth S, Islam W, Fang J, Šubr V, Röder B, Etrych T and Maeda H: Singlet oxygen phosphorescence detection in vivo identifies PDT-induced anoxia in solid tumors. Photochem Photobiol Sci 18: 1304-1314, 2019.

30. Dmitrieva VA, Tyutereva EV and Voitsekhovskaja OV: Singlet oxygen in plants: Generation, detection, and signaling roles. Int J Mol Sci 21: 3237, 2020.

31. Jantová S, Letasiova S, Brezova V, Cipak L and Labaj J: Photochemical and phototoxic activity of berberine on murine fibroblast NIH-3T3 and Ehrlich ascites carcinoma cells. J Photochem Photobiol B 85: 163-176, 2006.

32. Cheng L, Wang M, Zhao P, Zhu H, Zhu R, Sun X, Yao S and Wang S: The examination of berberine excited state by laser flash photolysis. Spectrochim Acta A Mol Biomol Spectrosc 73 268-272, 2009

33. Cheng LL, Wang M, Wu MH, Yao SD, Jiao Z and Wang SL: Interaction mechanism between berberine and the enzyme lysozyme. Spectrochim Acta A Mol Biomol Spectrosc 97: 209-214, 2012.

34. Shen L and Ji HF: The mechanisms of ROS-photogeneration by berberine, a natural isoquinoline alkaloid. J Photochem Photobiol B 99: 154-156, 2010.

35. Liu R and Zhang Y: Mechanism of UV-driven photoelectrocatalytic degradation of berberine chloride form using the ESR Spin-trapping method. Photochem Photobiol 94: 650-658, 2018.

36. Görner H, Miskolczy Z, Megyesi M and Biczok L: Photooxidation of alkaloids: Considerable quantum yield enhancement by rose bengal-sensitized singlet molecular oxygen generation. Photochem Photobiol 87: 1315-1320, 2011.

37. Görner H, Miskolczy Z, Megyesi M and Biczok L: Photoreduction and ketone-sensitized reduction of alkaloids. Photochem Photobiol 87: 284-291, 2011.
38. Hirakawa K, Kawanishi S and Hirano T: The mechanism of guanine specific photooxidation in the presence of berberine and palmatine: Activation of photosensitized singlet oxygen generation through DNA-binding interaction. Chem Res Toxicol 18: $1545-1552,2005$

39. Chen XW, Di YM, Zhang J, Zhou ZW, Li CG and Zhou SF: Interaction of herbal compounds with biological targets: A case study with berberine. ScientificWorldJournal 2012: 708292, 2012.

40. Hirakawa K and Hirano T: The microenvironment of DNA switches the activity of singlet oxygen generation photosensitized by berberine and palmatine. Photochem Photobiol 84: 202-208, 2008.

41. Hirakawa K, Hirano T, Nishimura Y, Arai T and Nosaka Y: Dynamics of singlet oxygen generation by DNA-binding photosensitizers. J Phys Chem B 116: 3037-3044, 2012.

42. Jiang D and Rusling JF: Oxidation chemistry of DNA and p53 tumor suppressor gene. ChemistryOpen 8: 252-265, 2019.

43. Cheng LL, Wang YJ, Huang DH, Yao SD, Ding GJ, Wang SL and Jiao Z: Radiolysis and photolysis studies on active transient species of berberine. Spectrochim Acta A Mol Biomol Spectrosc 124: 670-676, 2014.

44. Wu J, Xiao Q, Zhang N, Xue C, Leung AW, Zhang H, Tang QJ and $\mathrm{Xu} C$ : Palmatine hydrochloride mediated photodynamic inactivation of breast cancer MCF-7 cells: Effectiveness and mechanism of action. Photodiagnosis Photodyn Ther 15: 133-138, 2016.

45. Bhattacharyya R, Gupta P, Bandyopadhyay SK, Patro BS and Chattopadhyay S: Coralyne, a protoberberine alkaloid, causes robust photosenstization of cancer cells through ATR-p38 MAPK-BAX and JAK2-STAT1-BAX pathways. Chem Biol Interact 285: 27-39, 2018.

46. Wu J, Xiao Q, Zhang N, Xue C, Leung AW, Zhang H, Xu C and Tang QJ: Photodynamic action of palmatine hydrochloride on colon adenocarcinoma HT-29 cells. Photodiagnosis Photodyn Ther 15: 53-58, 2016.

47. Qi F, Sun Y, Lv M, Qin F, Cao W and Bi L: Effects of palmatine hydrochloride mediated photodynamic therapy on oral squamous cell carcinoma. Photochem Photobiol Sci 18: 1596-1605, 2019.

48. Patro BS, Bhattacharyya R, Gupta P, Bandyopadhyay S and Chattopadhyay S: Mechanism of coralyne-mediated DNA photo-nicking process. J Photochem Photobiol B 194: 140-148, 2019.

49. Ihmels $\mathrm{H}$ and Salbach A: Efficient photoinduced DNA damage by coralyne. Photochem Photobiol 82: 1572-1576, 2006.

50. Basu A, Jaisankar P and Kumar GS: Photophysical and calorimetric studies on the binding of 9-O-substituted analogs of the plant alkaloid berberine to double stranded poly(A). J Photochem Photobiol B 125: 105-114, 2013.

51. Basu A, Jaisankar P and Suresh Kumar G: Synthesis of novel 9-O-N-aryl/aryl-alkyl amino carbonyl methyl substituted berberine analogs and evaluation of DNA binding aspects. Bioorg Med Chem 20: 2498-2505, 2012.

52. Liu C, Liu S, Wang Y, Wang S, Zhang J, Li S, Qin X, Li X, Wang K and Zhou Q: Synthesis, cytotoxicity, and DNA-binding property of berberine derivatives. Med Chemistry Res 23: 1899-1907, 2014

53. Molero ML, Hazen MJ and Stockert JC: Photodynamic effect of berberine sulfate on the growth rate of allium cepa roots. J Plant Physiol 120: 91-94, 1985.

54. Lee NK, Jenner L, Harney A and Cameron J: Pharmacotherapy for amphetamine dependence: A systematic review. Drug Alcohol Depend 191: 309-337, 2018.

55. Lee MMS, Zheng L, Yu B, Xu W, Kwok RTK, Lam JWY, Xu F, Wang D and Tang BZ: A highly efficient and AIE-active theranostic agent from natural herbs. Materials Chemistry Front 3: 1454-1461, 2019.

56. Ma XQ, Liu HL, Cheng GP, Yuan SC and Liang B: Effects of berberine combined with photodynamic on apoptosis of gastric cancer MGC-803 Cell. Chin J Clin Pharmacol Therapeutics 20: 961-966, 2015.

57. Chen KT, Hao DM, Liu ZX, Chen YC and You ZS: Effect of berberine alone or in combination with argon ion laser treatment on 9L rat glioma cell line. Chin Med J (Engl) 107: 808-812, 1994

58. Lopes TZ, de Moraes FR, Tedesco AC, Arni RK, Rahal P and Calmon MF: Berberine associated photodynamic therapy promotes autophagy and apoptosis via ROS generation in renal carcinoma cells. Biomed Pharmacother 123: 109794, 2020. 
59. Warowicka A, Popenda $Ł$, Bartkowiak G, Musidlak O, Litowczenko-Cybulska J, Kuźma D, Nawrot R, Jurga S and Goździcka-Józefiak A: Protoberberine compounds extracted from Chelidonium majus L. as novel natural photosensitizers for cancer therapy. Phytomedicine 64: 152919, 2019.

60. Wang Y, Liu Y, Du X, Ma H and Yao J: The anti-cancer mechanisms of berberine: A review. Cancer Manag Res 12: 695-702, 2020.

61. Liu HQ, An YW, Li ZW, Li WX, Yuan B, Wang JC, Jin HT and Wang C: Sinoporphyrin sodium, a novel sensitizer for photodynamic and sonodynamic therapy. Open Chemistry 18: 691-701, 2020.

62. An YW, Liu HQ, Zhou ZQ, Wang JC, Jiang GY, Li ZW, Wang F and Jin HT: Sinoporphyrin sodium is a promising sensitizer for photodynamic and sonodynamic therapy in glioma. Oncol Rep 44: 1596-1604, 2020

63. Wang X, He L, Liu B and Wang J: Spectroscopic investigation on the sonodynamic damage to proteins in the presence of berberine in vitro. J Luminescence 131: 1361-1367, 2011

64. Geng C, Zhang Y, Hidru TH, Zhi L, Tao M, Zou L, Chen C, $\mathrm{Li} \mathrm{H}$ and Liu Y: Sonodynamic therapy: A potential treatment for atherosclerosis. Life Sci 207: 304-313, 2018.

65. Tian Y and Guo S: Sonodynamic effect of berberine on macrophages. Heart 98: E87-E88, 2012.

66. Kou JY, Li Y, Zhong ZY, Jiang YQ, Li XS, Han XB, Liu ZN, Tian Y and Yang LM: Berberine-sonodynamic therapy induces autophagy and lipid unloading in macrophage. Cell Death Dis 8 : e2558, 2017.

67. Sun HW and Ouyang WQ: Preparation and physicochemical characteristics of berberine hydrochloric nanoemulsion. Chin Traditional Herbal Drugs 38: 1476-1480, 2007.

68. Song J, Lin C, Yang X, Xie Y, Hu P, Li H, Zhu W and Hu H: Mitochondrial targeting nanodrugs self-assembled from 9-O-octadecyl substituted berberine derivative for cancer treatment by inducing mitochondrial apoptosis pathways. J Control Release 294: 27-42, 2019.

69. Fan JX, Liu MD, Li CX, Hong S Zheng DW, Liu XH, Chen S, Cheng $\mathrm{H}$ and Zhang XZ: A metal-semiconductor nanocomposite as an efficient oxygen-independent photosensitizer for photodynamic tumor therapy. Nanoscale Horiz 2: 349-355, 2017.

70. Wang C, Dai C, Hu Z, Li H, Yu L, Lin H, Bai J and Chen Y: Photonic cancer nanomedicine using the near infrared-II biowindow enabled by biocompatible titanium nitride nanoplatforms. Nanoscale Horiz 4: 415-425, 2019.

71. Zhen W, Liu Y, Jia X, Wu L, Wang C and Jiang X: Reductive surfactant-assisted one-step fabrication of a BiOI/BiOIO3 heterojunction biophotocatalyst for enhanced photodynamic theranostics overcoming tumor hypoxia. Nanoscale Horiz 4: 720-726, 2019

72. Zhang D, Zhang J, Li Q, Tian H, Zhang N, Li Z and Luan Y: pHand enzyme-sensitive IR820-paclitaxel conjugate self-assembled nanovehicles for near-infrared fluorescence imaging-guided chemo-photothermal therapy. ACS Appl Mater Interfaces 10: 30092-30102, 2018

73. Zhang J, Zhang D, Li Q, Jiang Y, Song A, Li Z and Luan Y: Task-specific design of immune-augmented nanoplatform to enable high-efficiency tumor immunotherapy. ACS Appl Mater Interfaces 11: 42904-42916, 2019.
74. Rajpoot K: Solid lipid nanoparticles: A promising nanomaterial in drug delivery. Curr Pharm Des 25: 3943-3959, 2019.

75. Hou J and Zhou SW: Optimization of the preparation technology of berberine hydrochloride solid lipid nanoparticles by orthogonal experiment. China Pharmacy 19: 1150-1152, 2008.

76. Wang Y, Zheng J, Xu B, Wang H, Deng Y and Bi D: Determination of entrapment efficiency of berberine hydrochloride solid lipid nanoparticles by coagulation-centrifuge method. J Zhengzhou Univ (Med Ences) 44: 188-189, 2009.

77. Elhissi A: Liposomes for pulmonary drug delivery: The role of formulation and inhalation device design. Curr Pharm Des 23: 362-372, 2017.

78. Luo X, Li J, Guo L, Cheng X, Zhang T and Deng Y: Preparation of berberine hydrochloride long-circulating liposomes by ionophore A23187-mediated ZnSO4 gradient method. Asian J Pharmaceutical Ences 8: 261-266, 2013.

79. Chen J, Tian L, Li W and Li G: Study on the preparation process of berberine hydrochloride liposomes by orthogonal design. J Practical Med Techniques 14: 1868-1870, 2007.

80. Kapoor DN, Bhatia A, Kaur R, Sharma R, Kaur G and Dhawan S: PLGA: A unique polymer for drug delivery. Ther Deliv 6: 41-58, 2015.

81. Khan I, Joshi G, Nakhate KT, Ajazuddin, Kumar R and Gupta U: Nano-Co-delivery of berberine and anticancer drug using PLGA nanoparticles: Exploration of better anticancer activity and in vivo kinetics. Pharm Res 36: 149, 2019.

82. Guo T, Fan Y, Chen M, Wu X, Zhang L, He T, Wang H, Wan J, Wang $\mathrm{X}$ and $\mathrm{Lu} \mathrm{Z}$ : Cardiovascular implications of fatal outcomes of patients with coronavirus disease 2019 (COVID-19). JAMA Cardiol 5: 811-818, 2020.

83. Guo HH, Feng CL, Zhang WX, Luo ZG, Zhang HJ, Zhang TT, Ma C, Zhan Y, Li R, Wu S, et al: Liver-target nanotechnology facilitates berberine to ameliorate cardio-metabolic diseases. Nat Commun 10: 1981, 2019.

84. Bhatnagar P, Kumari M, Pahuja R, Pant AB, Shukla Y, Kumar P and Gupta KC: Hyaluronic acid-grafted PLGA nanoparticles for the sustained delivery of berberine chloride for an efficient suppression of Ehrlich ascites tumors. Drug Deliv Transl Res 8: 565-579, 2018.

85. Grebinyk A, Prylutska S, Buchelnikov A, Tverdokhleb N, Grebinyk S, Evstigneev M, Matyshevska O, Cherepanov V, Prylutskyy $\mathrm{Y}$, Yashchuk V, et al: $\mathrm{C}_{60}$ fullerene as an effective nanoplatform of alkaloid berberine delivery into leukemic cells. Pharmaceutics 11: 586, 2019.

This work is licensed under a Creative Commons Attribution-NonCommercial-NoDerivatives 4.0 International (CC BY-NC-ND 4.0) License. 